\title{
Diaphragm pacing restores olfaction in tetraplegia
}

\author{
D. Adler*,\#, J. Gonzalez-Bermejo*,\#, A. Duguet*,\#, A. Demoule*,\#, \\ F. Le Pimpec-Barthes ${ }^{+}$, A. Hurbault*, C. Morélot-Panzini, ${ }^{\star, *}$ and T. Similowski*, ${ }^{*}$
}

ABSTRACT: High cervical spinal cord injuries induce extreme handicap and tactile isolation. Tracheotomised tetraplegic patients are also bound to be olfaction deprived. By restoring negative pressure inspiration, diaphragm pacing (DP) should improve olfaction.

We tested olfaction in 10 consecutive tetraplegics during positive pressure mechanical ventilation and DP, using the University of Pennsylvania Smell Identification Test (UPSIT). Quality of life was assessed using the Satisfaction with Life Scale (SWLS). Self-perceived benefits of DP were studied using an in-house questionnaire.

Olfaction was very poor during positive pressure mechanical ventilation (UPSIT, mean \pm SD $17.1 \pm 6.4$, anosmia or severe microsmia). It improved during DP $(35.2 \pm 1.9$, normosmia or mild microsmia; $p<0.0001$ ) and SWLS was $18.5 \pm$ 4.2. Nine patients stated that DP had improved their quality of life. This was driven by better mobility (ranked first), improved self-image and relationships with others (ranked second), improved olfaction and better feeling of security (both ranked third).

Improved olfaction is among the benefits of DP and should be mentioned to patients considered for this therapy. Furthermore, attention to olfaction is warranted in tracheotomised ventilatordependent patients, as a putative path towards improvement of quality of life.

KEYWORDS: Phrenic stimulation, quality of life, spinal cord injury, tracheotomy, ventilator-dependency

$\mathrm{T}$ raumatic spinal cord injury $(\mathrm{SCI})$ disrupts body image, disorganises daily life and shatters life plans. Cervical lesions responsible for tetraplegia produce considerable disability capped by near complete tactile isolation. In tracheotomised ventilator-dependent patients, speech difficulties worsen this isolation. This brings about the feelings of helplessness and uselessness commonly reported early after the trauma [1]. Self-perceived quality of life is initially low, although it improves over time [2,3].

In addition to tactile isolation, tetraplegic, tracheotomised and mechanically ventilated patients are also bound to be deprived of the sense of smell. Indeed, they cannot only poorly inspire but their nasal mucosa is bypassed from the inspiratory flux, thus inaccessible to odorant molecules. Olfactory impairment and the related alteration in their sense of taste [4] would deny these patients from one of their few remaining sources of physical satisfaction.

Diaphragm pacing has been proposed to wean tetraplegics from mechanical ventilation $[5,6]$. The corresponding negative pressure driven inspiratory flow should re-irrigate the nasal fossae and improve olfaction. This has been mentioned in some reviews on diaphragm pacing [7-9], but never studied in a systematic manner. Of note, olfaction is not mentioned in the recent and only controlled study on diaphragm pacing [6].

We hypothesised that: 1) tracheotomised and mechanically ventilated tetraplegic patients would have poor olfactory performances; 2) olfactory performances would improve with diaphragm pacing; and 3) this would be perceived as a benefit of diaphragm pacing. To test this hypothesis, we conducted standardised olfactory tests in tetraplegic patients with respiratory neurostimulators during diaphragm pacing and during mechanical ventilation. In addition, we tried to evaluate how important the recovery of olfaction stood in the perspective of selfevaluation of diaphragm pacing-derived benefits.

\section{PATIENTS AND METHODS \\ Patients}

We studied 10 consecutive tetraplegic patients (table 1) with intrathoracic phrenic nerve stimulation $\left(n=9 ;\right.$ Atrostim $^{\mathrm{TM}}$; Atrotech, Tampere,
AFFILIATIONS

*APHP, Service de Pneumologie et Réanimation et Centre de Stimulation Phrénique Implantée, Groupe Hospitalier Pitié-Salpêtrière, ${ }^{+}$APHP, Service de Chirurgie Thoracique, Hôpital Européen Georges Pompidou,

"Université Paris 6, ER 10 UPMC Paris, France, and

'Service de Pneumologie, Hôpital Cantonal Universitaire de Genève Geneva, Switzerland.

CORRESPONDENCE T. Similowski

Service de Pneumologie et Réanimation

Groupe Hospitalier Pitié-Salpêtrière 47-83 Bd de l'Hôpital 75651 Paris

Cedex 13

France

E-mail: thomas.similowski@ psl.ap-hop-paris.fr

Received: Nov 232008 Accepted after revision: Feb 032009 First published online: Feb 272009 
Finland) or intradiaphragmatic pacing $\left(n=1\right.$; NeuRXDPS ${ }^{\mathrm{TM}}$; Synapse Biomedical, Oberlin, OH, USA). The patients were studied during a programmed routine visit. They were clinically stable, free of ear, nose and throat symptoms, had never smoked or had not smoked since the onset of tetraplegia, and did not report past olfactory problems. They were all tracheotomised and were studied with uncuffed tracheotomy cannulae or uninflated cannulae cuffs. All the patients had normal scores on the Mini-Mental State Examination; manual tasks were excluded [10].

The study was approved by the Institutional Review board of the French Learned Society for Intensive Care Medicine (Société de Réanimation de Langue Française, Paris, France) and informed consent was systematically obtained.

Of note, since 1996, 24 patients have been implanted with neurostimulators at the French national centre for phrenic stimulation (Paris). Of these, 15 were alive at the time of the present study, five of whom were not included because of pacing failure $(n=1)$, severe depression $(n=1)$ or cognitive defects $(n=3)$.

\section{Olfaction}

\section{Smell identification test}

Olfactory performances were evaluated using the French version of the University of Pennsylvania Smell Identification Test (UPSIT; Sensonics ${ }^{\circledR}$, Haddon Heights, NJ, USA) [11], which is easy to use and has a high test-retest reliability [12]. The test consists of four 10-page booklets, each page containing a microcapsule that disperses an odorant molecule when scratched. For each odorant, the subject is asked to choose one identifier among four propositions immediately after the microcapsule has been scratched. A response is required even if no smell is perceived (forced choice). Once the four booklets, presented in random order, have been completely used, the patient's score is determined. It is compared to normative values derived from a database of several thousand normal individuals [11]. Any patient can be allocated a percentile rank corresponding to their age and sex group, or categorised as has having normosmia, mild microsmia, moderate microsmia, severe miscosmia or anosmia.

\section{Protocol}

The UPSIT was performed over a period of $\sim 20 \mathrm{~min}$ on two consecutive mornings in random order: 1) during positive pressure mechanical ventilation with neurostimulation switched off; and 2) during diaphragm pacing with the ventilator disconnected. In both cases, the tracheotomy was open, and the ventilation settings were those normally used by the patients, providing similar levels of ventilation. The odorants were presented in front of the patients' noses. The patients were instructed not to perform any kind of sniffing manoeuvres.

General evaluation of quality of life

The patients self-rated their quality of life using the Satisfaction with Life Scale (SWLS) [13], a five-item instrument designed to measure global cognitive judgments of one's life, which has previously been used in SCI patients $[3,14]$.

\section{Self-perceived impact of diaphragm pacing on daily life}

At the time of olfaction testing, the opinion of the patients regarding respiratory neurostimulation was sought using a specifically designed 17-item self-administered questionnaire. The questionnaire included nine questions pertaining to quality of life: How did diaphragm pacing affect your,

\section{TABLE 1 Characteristics of the patients}

\begin{tabular}{|c|c|c|c|c|c|c|c|c|}
\hline Patient & Sex & Age yrs & \multicolumn{2}{|c|}{ Spinal injury } & Time since injury yrs & Time since DP yrs & Place of residence & SWLS score ${ }^{+}$ \\
\hline 2 & M & 26 & C3 & Post-op ${ }^{f}$ & 1.5 & 1 & Institution & 17 \\
\hline 3 & M & 53 & $\mathrm{C} 1$ & Traumatic & 7.9 & 6 & Home & 23 \\
\hline 4 & M & 30 & $\mathrm{C} 2 \mathrm{C} 3$ & Traumatic & 15.7 & 14 & Home & 17 \\
\hline 6 & M & 23 & $\mathrm{C} 1 \mathrm{C} 2$ & Traumatic & 4.3 & 4 & Home & 19 \\
\hline 7 & $\mathrm{~F}$ & 61 & C1 & Post-op ${ }^{f}$ & 1 & 0.1 & Hospital & 21 \\
\hline 8 & M & 52 & $\mathrm{C} 2$ & Traumatic & 18.3 & 17 & Institution & 16 \\
\hline 9 & M & 45 & $\mathrm{C} 2$ & Traumatic & 10.9 & 10.1 & Home & 26 \\
\hline 10 & $\mathrm{~F}$ & 21 & $\mathrm{C} 2$ & Traumatic & 4 & 3 & Home & 16 \\
\hline Mean $\pm S D^{\S}$ & & $35.8 \pm 15.3$ & & & $7.05 \pm 6.03$ & $6.0 \pm 5.9$ & & $18.5 \pm 4.2$ \\
\hline
\end{tabular}

DP: diaphragm pacing; SWLS: satisfaction with life scale; M: male; F: female; Op: operative. \# : consolidated neurological level; ": at the time of testing. ${ }^{+}$: comprises five items: 1) in most ways my life is close to my ideal; 2) the conditions of my life are excellent; 3) I am satisfied with my life; 4) so far I have gotten the important things I want in life; 5) if I could live my life over, I would change almost nothing. Each statement is scored on a seven-point Likert scale: $7=$ strongly agree; $6=$ agree; $5=$ slightly agree; $4=$ neither agree nor disagree; 3 =slightly disagree; 2 =disagree; $1=$ strongly disagree. The final score allocates the patient to one of seven categories. $35-31$ : extremely satisfied; 26-30: satisfied; 21-25: slightly satisfied; 20: neutral; 15-19: slightly dissatisfied; 10-14: dissatisfied; 5-9: extremely dissatisfied. ${ }^{\text {s: }}$ all data sets passed the Shapiro-Wilk normality test; ${ }^{f}$ : neurosurgery (ependymoma). 
1: overall quality of life; 2: body image; 3: schooling/ professional life; 4: leisure activities; 5: sexual life; 6: relationships with others; 7: ability to get out of your bed; 8 : ability to move out of your home; and 9: quality of sleep? Five questions pertained to sensory performances: How did diaphragm pacing affect your, 10: sense of smell; 11: sense of taste; 12: auditory environment; 13: sight; and 14: sense of touch? The latter two questions were intended as "controls". The questionnaire also included one question on the impact of an improved sense of smell on quality of life: 15: How did a change in your sense of smell affect your quality of life after diaphragm pacing? Finally, two questions explored the general perception of respiratory neurostimulation: 16: Would you agree to return to your previous ventilatory mode? and 17: Would you recommend diaphragm pacing to others? To avoid grouping effects, questions from the above groups were alternated. Each statement was scored on a five-point Likert scale (-2: much deteriorated; -1: deteriorated; 0: no change; 1: improved; 2 : much improved).

Patients were interviewed by telephone or e-mail in an open manner $\geqslant 6$ months after the initial study and asked to provide an unrestricted list of what they considered as benefits of diaphragm pacing, ranked by decreasing order of importance. They were also asked, when relevant, why they found that an improved olfaction was the source of improved quality of life.

\section{Statistical analysis}

Statistical testing was performed using Prism4 ${ }_{\circledR}$ (GraphPad, San Diego, CA, USA) and StatXact ${ }_{\circledast}$ (Cytel, Cambridge, MA, USA).

All the data distributions were normal according to the Schapiro-Wilk test, the results are expressed as mean \pm SD. Differences were considered significant when the probability of a type I error was $p \leqslant 0.05$. Olfactory performances were compared between the two ventilatory modes using a paired t-test. The p-values are reported with indication of the $t$ statistics ( $t$ ) and of the degrees of freedom (df). The distribution of the answers along the five-point Likert scale used in the questionnaire was studied from $5 \times 2$ contingency

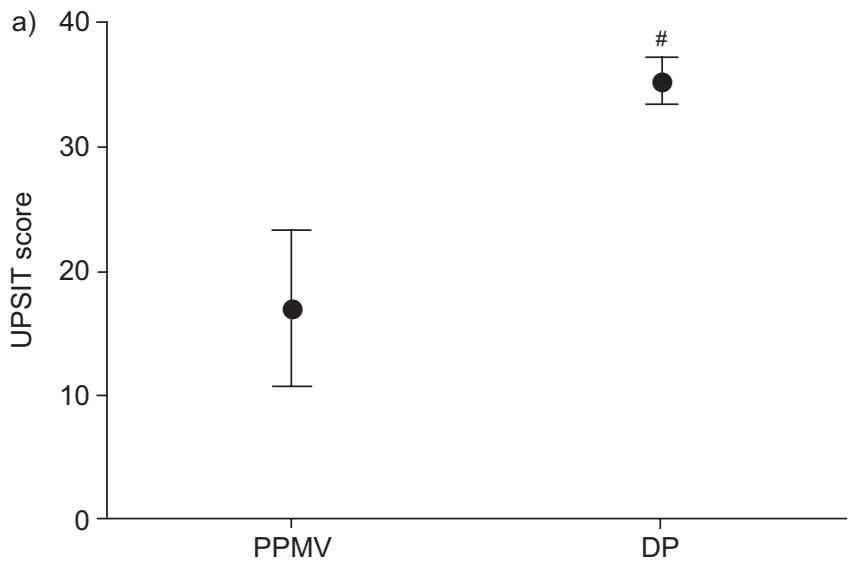

tables with Fisher's exact test. When the existence of a significant difference was detected, the positive answers (1 and 2 ) and the neutral and negative answers (0, -1, -2) were grouped to construct $2 \times 2$ contingency tables to which the Fisher's test was again applied.

\section{RESULTS}

\section{Olfaction}

Of note, chart reviews showed that all but one patient had spontaneously reported a qualitative improvement in their sense of smell with diaphragm pacing soon after its introduction.

During positive pressure mechanical ventilation, the UPSIT score was $17.1 \pm 6.4$ (9-27=anosmia or severe microsmia). During diaphragm pacing, the UPSIT score rose to $35.2 \pm 1.9$ (33-38=normosmia or mild microsmia) (fig. 1). The difference was highly significant $(p<0.0001, t=9.167, d f=9)$. All the patients considered that diaphragm pacing had "improved" or "much improved" their sense of smell and six reported an improved sense of taste (fig. 2).

\section{Quality of life}

The SWLS score was $18.5 \pm 4.2$ (table 1 ).

\section{Self-perceived impact of diaphragm pacing on daily life}

Figure 2 summarises the results of the diaphragm pacing questionnaire. Of particular interest: 1) nine patients reported that diaphragm pacing had improved their overall quality of life; 2) the patients were unanimous in not considering any return to their previous ventilatory assistance mode and in being willing to recommend diaphragm pacing to other patients; and 3) nine patients considered that the improvement in olfaction associated with diaphragm pacing had improved $(n=6)$ or much improved $(n=3)$ their quality of life. Nine patients answered the post hoc interviews. They all ranked improved mobility and sense of freedom (or words to that effect) as the main benefit of diaphragm pacing. Improved body image and better relationships with others came second (ranked second in five patients, third in three and fourth in one

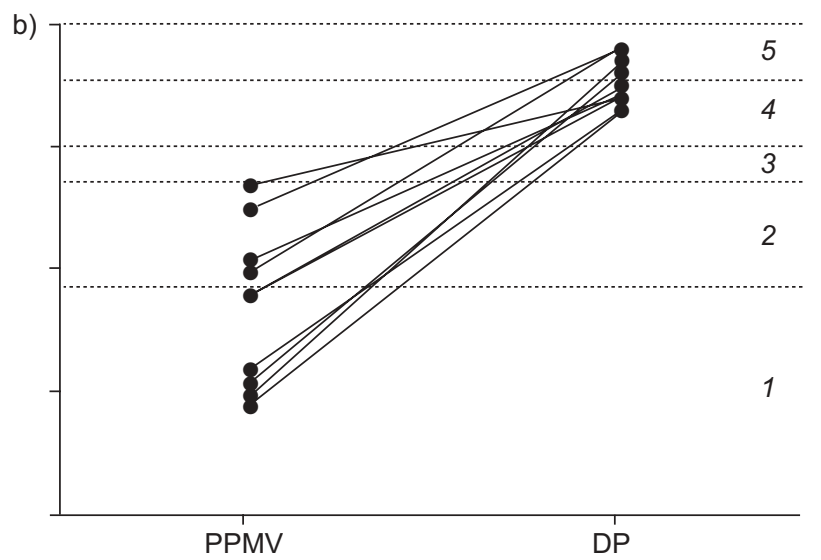

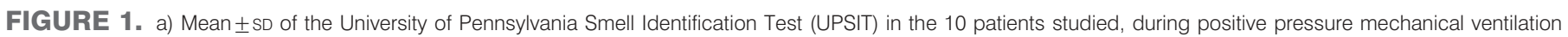

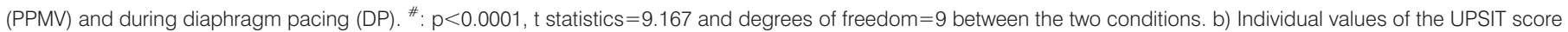

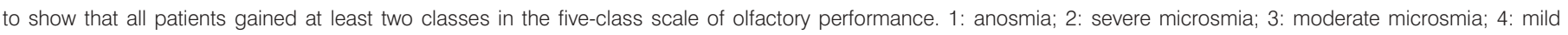
microsmia; and 5: normosmia. 
a) $10-1$. How did diaphragm pacing affect your overall quality of life?

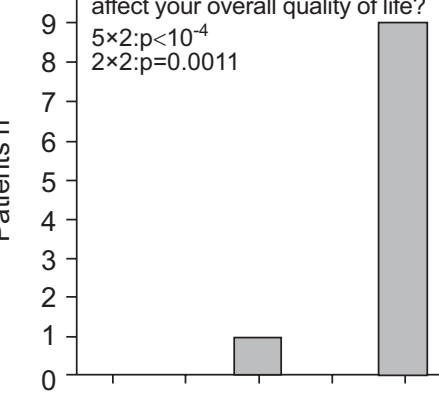

e) 10$\urcorner 5$. How did diaphragm pacing

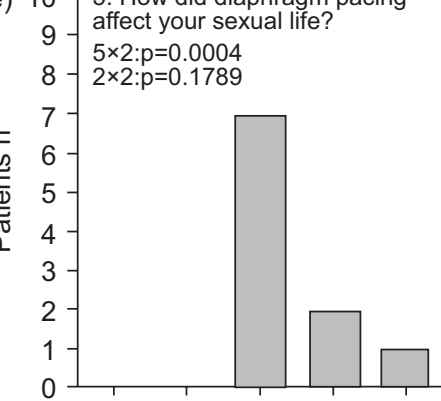

i) $10-9$. How did diaphragm pacing affect your quality of sleep?
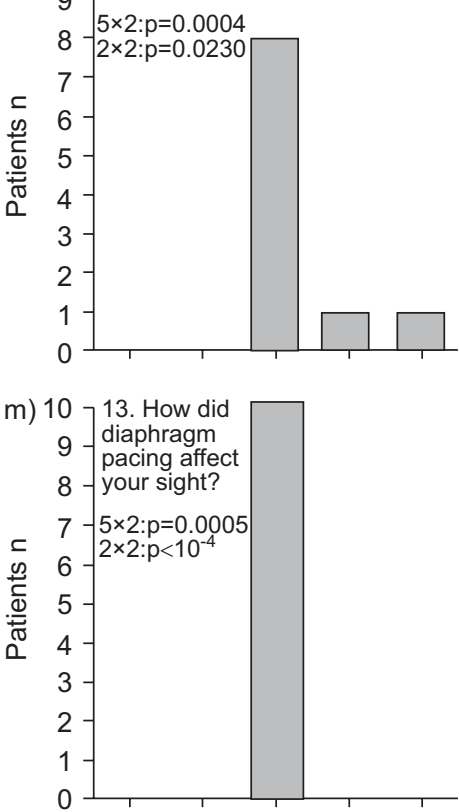

q) $10-17$. Would you

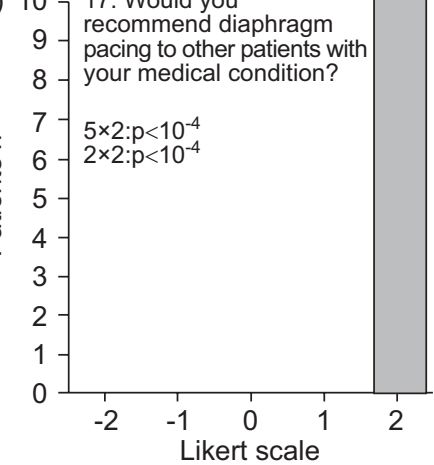

b) 2. How did diaphragm pacing affect c) your body image?

$5 \times 2: p=0.0055$

$2 \times 2: p=0.0230$

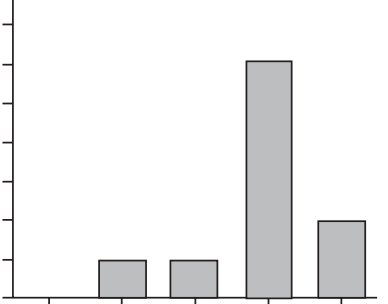

f) 7 . How did diaphragm pacing affect your relationships with others (family, friends)?

$5 \times 2: p<10^{-4}$

$2 \times 2: p<10^{-4}$

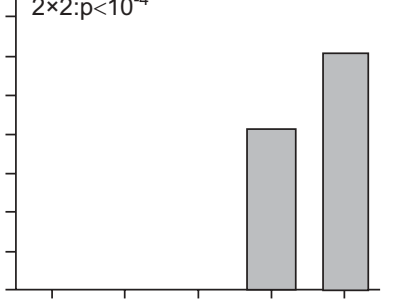

j) 10. How did diaphragm pacing affect $k$ your sense of smell?

$5 \times 2: p=0.0005$

$2 \times 2: p<10^{-4}$

$-$

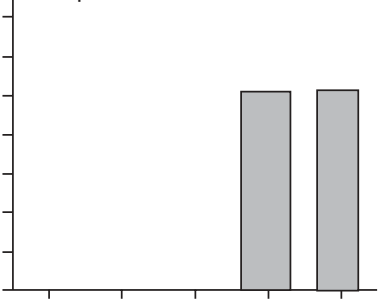

n)

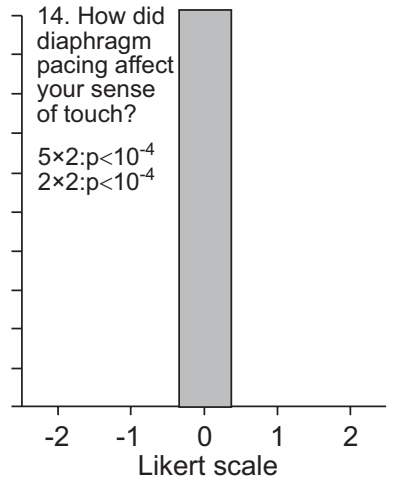

3. How did diaphragm pacing affect your schooling or professional life? $5 \times 2: p=0.0065$ $2 \times 2: p=1$

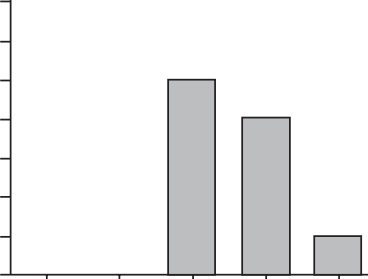

7. How did diaphragm pacing affect your ability to get out of bed?

$-5 \times 2: p=0.0004$ $2 \times 2: p=0.0004$

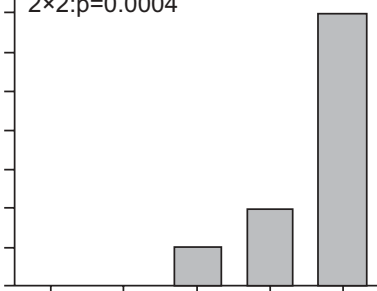

11. How did diaphragm pacing - affect your sense of taste?

$5 \times 2: p=0.0258$ $2 \times 2: p=0.7787$

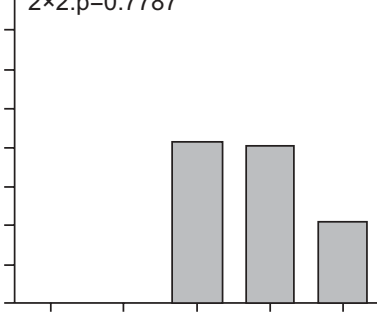

o)

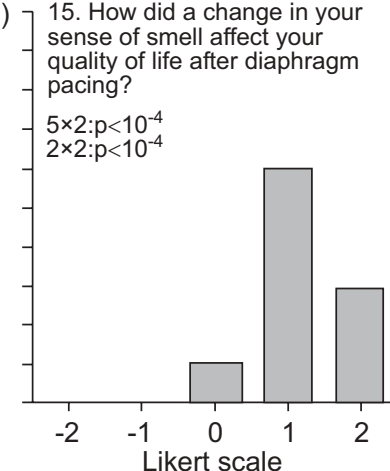

d) 4. How did diaphragm pacing affect your leisure activities? $5 \times 2: p=0.0022$ $2 \times 2: p=0.0011$

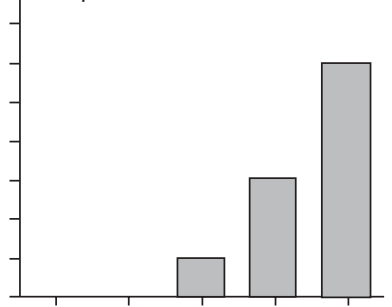

h) 8. How did diaphragm pacing affect your mobility outside your home? $5 \times 2: p=0.0004$ $2 \times 2: p=0.0230$

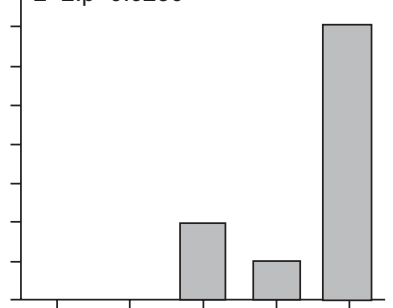

I) 12. How did diaphragm pacing affect your lauditory environment? $5 \times 2: p=0.0258$ $2 \times 2: p=0.7787$

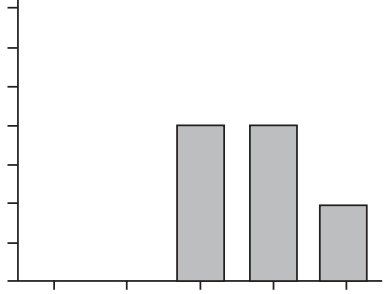

p

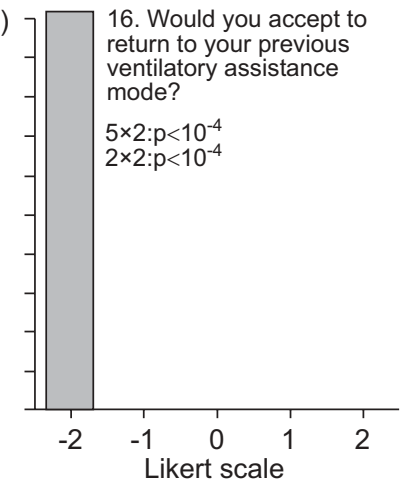

FIGURE 2. Distribution of the answers provided by the 10 patients to the 17 -item diaphragm pacing oriented questionnaire. a-i) Questions 1 to 9 pertain to quality of life. j-o) Questions 10 to 15 pertain to sensory perceptions. Questions 14 and 15 are intended as "controls", with an answer of "0" expected. p, q) Questions 16 and 17 explore the general opinion of the patients about diaphragm pacing. a-0) The Likert scale read: -2: much deteriorated; -1 : deteriorated; 0 : no change; 1: improved; 2: much improved. p, q) Likert scale read: -2: completely disagree; -1: disagree; 0: no change; 1 : agree; 2 : completely agree. The questions are grouped by theme but were alternated when asked. 
patient), followed by improved olfaction (ranked second in one patients third in four and fourth in four patients) which was evenly ranked with improved sense of security (second in two patients, third in two and fourth in five patients). This was followed by improved speech, as mentioned by six patients. An improved sense of taste and better sleep were mentioned by only five and three patients, respectively, and then ranked last or penultimate. The improved olfaction was valued by all the patients for two reasons: 1) better perception of the environment, and 2) more pleasure with food.

\section{DISCUSSION}

Since the start of diaphragm pacing at the French national centre for phrenic stimulation in 1996, almost all our patients have spontaneously reported, and valued, olfactive improvements with diaphragm pacing, with some striking anecdotal reports (e.g. the capacity of a professional oenologist to resume work after diaphragm pacing). Therefore, we conducted this study to: 1) show this clinical impression; 2) quantify the magnitude of the olfaction improvement; and 3) gain an insight in the putative effects of improved olfaction on quality of life. Our results document that olfaction is severely impaired in tracheotomised ventilator-dependent tetraplegic patients and that it is indeed improved by diaphragm pacing. In a less predictable manner, our results also show that diaphragm pacing is sufficient to bring the olfactory identification capacity back to normal in this setting, and the results document that this may contribute to a better quality of life.

\section{Mechanisms of improved olfaction}

Our study supports the fact that olfaction was altered in our patients simply because their nasal fossae was bypassed during positive pressure ventilation on tracheostomy. Diaphragm pacing corrected it through the re-irrigation of the nose by room air.

A negative impact of tracheostomy on olfaction has been previously described [15-18]. LICHTMAN et al. [16] reported low odorant identification scores in six tracheotomised patients; a Passy-Muir speaking valve improved performances. Olfactory testing was performed by holding small pieces of odorantsoaked blotting paper in front of the patient's tracheal opening, which was equipped with a speaking valve. By decreasing the amount of "perfumed" inhaled air leaving the airway through the tracheotomy during expiration, the speaking valve probably favoured retrograde irrigation of the nasal fossae and improved retronasal olfactory ability [19]. In contrast, in our patients, the improved smell-identification performances probably resulted from enhanced orthonasal olfactory abilities. Indeed, the odorant stimulations were presented in front of the noses of the patients, and the diaphragm pacing-induced aspiration necessarily resulted in an anterograde irrigation of the nasal fossae. Tracheotomy orifices were left open during olfactory testing under diaphragm pacing, to reproduce reallife conditions. Therefore, partial nasal re-irrigation is sufficient to restore near-normal olfaction (fig. 1). We acknowledge that we only studied the olfactory identification capacity and neglected olfactory thresholds and discrimination capacity. Nevertheless, we feel that our results are straightforward enough to give them clinical relevance.

\section{Quality of life}

On average, our patients had low SWLS scores. These scores were lower than those recently reported in tetraplegics [3], but the two populations are very different. All our patients were ventilator dependent. They were studied, on average, 7 yrs after injury versus 27.8 yrs after injury in the study by ABRANTES-PAIS FDE [3]. However, in agreement with known data [14], the SWLS scores tended to be higher in our patients who had the longest time since injury and who had been able to return home (table 1 ).

\section{Self-perceived impact of diaphragm pacing on daily life}

Our diaphragm-pacing questionnaire was not intended to measure treatment-induced changes in quality of life, in the absence of a control intervention that would be very difficult to conceive, and it has not been externally validated. Therefore, we acknowledge that it must be interpreted with caution. This is all the more the case that the questionnaire was applied to the patients in the cohort who were capable of answering it and in whom diaphragm pacing was effective; hence inherent selection and "success" biases. However, its design relied on a 10 -yr experience of the respiratory care of tetraplegic patients, in a large cohort of patients, where the results are in line with those reported by others regarding the patients' view of phrenic stimulation [6-9]. We therefore feel that the information provided is of clinical value. Of importance, the patients almost unanimously reported improvement in their perceived qualify of life with diaphragm pacing (fig. 2), even when they had low SWLS scores.

Our patients reported several changes in daily life as benefits of phrenic pacing. These benefits (improved mobility, better self-image and relationships with others, improved feeling of security, and better olfaction, etc.) are in line with previous reports [6-9]. Improved olfaction appears to contribute to the improved quality of life, with a "pleasure" dimension that is noteworthy in this setting. This is consistent with data showing that ameliorating impaired olfaction in laryngectomised patients, who also have bypassed nasal fossae, ameliorates quality of life [20].

\section{Conclusions and perspectives}

As hypothesised, olfaction is poor in mechanically ventilated patients, but it is improved by diaphragm pacing and it contributes to a better perception of quality of life. These observations are important to bear in mind when discussing diaphragm pacing with potential candidates. In addition, weight gain is a frequent problem in tetraplegic patients, and there are complex interactions between olfaction and appetite; anosmia is often associated with loss of appetite but the opposite is also possible [21]. In this view, our results could open new research perspectives regarding the nutritional management of these patients.

Beyond the very limited population dealt with by this study, these results are a strong incentive to take an interest in olfaction in other types of tracheotomised and long-term ventilator-dependent individuals. It could indeed be a path worthy of exploration to improve their quality of life, in the same perspective that has led, over the years, to speech improvement measures in these patients $[22,23]$. The impact of long-term noninvasive ventilation on olfaction should also be 
studied because of the local effects that this ventilatory mode has, at times, on the nasal mucosa.

\section{SUPPORT STATEMENT}

D. Adler was supported by Fédération ANTADIR (Paris, France) and the Fonds de Perfectionnement des Chefs de Clinique des Hôpitaux de Genève (Geneva, Switzerland). The study was supported by grant DRC98075 from the Programme Hospitalier de Recherche CliniqueNational (French Ministry of Health) and by Association pour le Développement et l'Organisation de le Recherche En Pneumologie (Paris). The diaphragm pacing activity of the Service de Pneumologie et Réanimation, Groupe Hospitalier Pitié-Salpêtrière (Paris) is supported in part by the Association d'Entraide des Polios et Handicapés (Puteaux, France).

\section{STATEMENT OF INTEREST}

A statement of interest for T. Similowski can be found at www.erj. ersjournals.com $/ \mathrm{misc} /$ statements.dtl

\section{ACKNOWLEDGEMENTS}

We would like to thank G. de Revel of the Université d'Oenologie (Bordeaux, France) and G. Sicard from the Centre des Sciences du Goût (Dijon, France), for their positive reactivity to the early phase of the project. We are also grateful to M. Amouyal-Jones for her help with English style and grammar.

\section{REFERENCES}

1 Hammell KW. Quality of life among people with high spinal cord injury living in the community. Spinal Cord 2004; 42: 607-620.

2 Westgren N, Levi R. Quality of life and traumatic spinal cord injury. Arch Phys Med Rehabil 1998; 79: 1433-1439.

3 Abrantes-Pais Fde N, Friedman JK, Lovallo WR, et al. Psychological or physiological: why are tetraplegic patients content? Neurology 2007; 69: 261-267.

4 Deems DA, Doty RL, Settle RG, et al. Smell and taste disorders, a study of 750 patients from the University of Pennsylvania Smell and Taste Center. Arch Otolaryngol Head Neck Surg 1991; 117: 519-528.

5 Glenn WW, Holcomb WG, Shaw RK, et al. Long-term ventilatory support by diaphragm pacing in quadriplegia. Ann Surg 1976; 183: 566-577.

6 Hirschfeld S, Exner G, Luukkaala T, et al. Mechanical ventilation or phrenic nerve stimulation for treatment of spinal cord injuryinduced respiratory insufficiency. Spinal Cord 2008; 46: 738-742.

7 Creasey G, Elefteriades J, DiMarco A, et al. Electrical stimulation to restore respiration. J Rehabil Res Dev 1996; 33: 123-132.
8 DiMarco AF. Restoration of respiratory muscle function following spinal cord injury. Review of electrical and magnetic stimulation techniques. Respir Physiol Neurobiol 2005; 147: 273-287.

9 DiMarco AF. Diaphragmatic pacing. In: Tobin MJ, ed. Principles and Practice of Mechanical Ventilation. 2nd Edn. New York, McGraw Hill Professional, 2006; pp 1263-1275.

10 Crum RM, Anthony JC, Bassett SS, et al. Population-based norms for the Mini-Mental State Examination by age and educational level. JAMA 1993; 269: 2386-2391.

11 Doty RL, Shaman P, Dann M. Development of the University of Pennsylvania Smell Identification Test: a standardized microencapsulated test of olfactory function. Physiol Behav 1984; 32: 489-502.

12 Doty RL, Frye RE, Agrawal U. Internal consistency reliability of the fractionated and whole University of Pennsylvania Smell Identification Test. Percept Psychophys 1989; 45: 381-384.

13 Diener E, Emmons RA, Larsen RJ, et al. The Satisfaction With Life Scale. J Pers Assess 1985; 49: 71-75.

14 Dijkers MP. Correlates of life satisfaction among persons with spinal cord injury. Arch Phys Med Rehabil 1999; 80: 867-876.

15 Rothschild MA, Myer CM 3rd., Duncan HJ., Olfactory disturbance in pediatric tracheotomy. Otolaryngol Head Neck Surg 1995; 113: 71-76.

16 Lichtman SW, Birnbaum IL, Sanfilippo MR, et al. Effect of a tracheostomy speaking valve on secretions, arterial oxygenation, and olfaction: a quantitative evaluation. J Speech Hear Res 1995; 38: 549-555.

17 Manzano JL, Lubillo S, Henriquez D, et al. Verbal communication of ventilator-dependent patients. Crit Care Med 1993; 21: 512-517.

18 Passy V, Baydur A, Prentice W, et al. Passy-Muir tracheostomy speaking valve on ventilator-dependent patients. Laryngoscope 1993; 103: 653-658.

19 Leon EA, Catalanotto FA, Werning JW. Retronasal and orthonasal olfactory ability after laryngectomy. Arch Otolaryngol Head Neck Surg 2007; 133: 32-36.

20 Risberg-Berlin B, Moller RY, Finizia C. Effectiveness of olfactory rehabilitation with the nasal airflow-inducing maneuver after total laryngectomy: one-year follow-up study. Arch Otolaryngol Head Neck Surg 2007; 133: 650-654.

21 Hirsch AR, Gomez R. Weight reduction through inhalation of odorants. J Neurol Orthop Med Surg 1995; 16: 28-31.

22 Hoit JD, Banzett RB, Lohmeier HL, et al. Clinical ventilator adjustments that improve speech. Chest 2003; 124: 1512-1521.

23 Prigent H, Samuel C, Louis B, et al. Comparative effects of two ventilatory modes on speech in tracheostomized patients with neuromuscular disease. Am J Respir Crit Care Med 2003; 167: 114-119. 